\title{
Nanotheranostics
}

2022; 6(1): 1-9. doi: 10.7150/ntno.58965

Research Paper

\section{Design and evaluation of Raman reporters for the Raman-silent region}

\author{
Konstantinos Plakas ${ }^{1 *}$, Lauren E. Rosch ${ }^{*}$, Michael D. Clark1, Shukree Adbul-Rashed1, Travis M. Shaffer ${ }^{2,3}$, \\ Stefan Harmsen $2,3,4, \bowtie$, Sanjiv S. Gambhir $2,3,5,6,{ }^{*}, t$, Michael R. Detty ${ }^{1, *}, \neq$ \\ 1. Department of Chemistry, University at Buffalo, The State University of New York, Buffalo, NY, USA. \\ 2. Molecular Imaging Program at Stanford University (MIPS), Stanford University School of Medicine, Stanford, CA, USA. \\ 3. Department of Radiology, Stanford University School of Medicine, Stanford, CA, USA. \\ 4. Department of Radiology, Perelman School of Medicine, University of Pennsylvania, PA, USA. \\ 5. Department of Bioengineering, Stanford University School of Medicine, Stanford, CA, USA. \\ 6. Department of Material Science \& Engineering, Stanford University School of Engineering, Stanford, CA, USA. \\ *Authors contributed equally. \\ ${ }^{\dagger}$ Deceased July 18, 2020 \\ ¥Deceased July 1, 2020 \\ $\triangle$ Corresponding author: Stefan Harmsen, PhD. Email: stefan.harmsen@pennmedicine.upenn.edu \\ (c) The author(s). This is an open access article distributed under the terms of the Creative Commons Attribution License (https://creativecommons.org/licenses/by/4.0/). \\ See http://ivyspring.com/terms for full terms and conditions.
}

Received: 2021.02.02; Accepted: 2021.03.08; Published: 2022.01.01

\begin{abstract}
Rationale: Surface enhanced Raman scattering (SERS) is proving to be a useful tool for biomedical imaging. However, this imaging technique can suffer from poor signal-to-noise ratio, as the complexity of biological tissues can lead to overlapping of Raman bands from tissues and the Raman reporter molecule utilized. Methods: Herein we describe the synthesis of triple bond containing Raman reporters that scatter light in the biological silent window, between $1750 \mathrm{~cm}^{-1}$ and $2750 \mathrm{~cm}^{-1}$. Results: Our SERS nanoprobes are comprised of uniquely designed Raman reporters containing either alkyne- or cyano-functional groups, enabling them to be readily distinguished from background biological tissue. Conclusion: We identify promising candidates that eventually can be moved forward as Raman reporters in SERS nanoparticles for highly specific contrast-enhanced Raman-based disease or analyte detection in biological applications.
\end{abstract}

Key words: Nanotag, surface-enhanced Raman scattering, Raman-silent region, triple bonds

\section{Introduction}

Surface-enhanced Raman scattering (SERS) has emerged as a highly promising bioanalytical and biomedical imaging platform. SERS is based on amplification of the Raman scattering cross-section of analytes adsorbed on noble-metal (e.g., gold) nanoparticle surfaces [1]. Furthermore, the fingerprint-like Raman spectra, which are a reflection of the vibrational transitions of the analyte, enable tremendous multiplex capabilities - simultaneous detection of up to 10 different SERS nanotags has been described in living subjects [2]. As such, SERS has not only been used to improve the sensitivity of (multi)analyte detection in bioanalytical assays [3-5], but to generate highly sensitive (targeted) Raman imaging probes for early and comprehensive disease detection in vivo [6-8].

To achieve the reported femto- to attomolar limits of detection of these reporter probes, analytes (or Raman reporters) that are resonant with the excitation laser were adsorbed on gold nanoparticles. Typically, these reporters are cyanine- or pyrylium-dyes because the electronic transitions of these dyes can be tuned to match with excitation wavelength of the widely used 785-nm lasers. However, since the Raman fingerprints of cyanine-dyes are complex, and, the fingerprints of 
pyrylium dyes demonstrate strong overlaps, this limits the multiplexing capabilities of SERS nanoprobes generated from (resonant) Raman reporters of these particular dye classes [9-12]. In order to expand the multiplexing capabilities of these classes of dyes and avoid spectral interferences, we sought to optimize the pyrylium dye structures for performance in the Raman silent region (1750-2750 $\mathrm{cm}^{-1}$ ) - a spectral window where Raman bands from biological molecules and tissues are minimized [13, 14] as only Raman bands from triple bond (e.g., alkyne, cyanide, nitrile, etc.) vibrational modes appear $[15,16]$. Since the Raman shift is relative to the energy of the excitation source, this Raman-silent window corresponds to a wavelength range of about 720-775 $\mathrm{nm}$ when the excitation wavelength is $638-\mathrm{nm}$ and to a range of 910-1000 $\mathrm{nm}$ for a 785-nm excitation source.

Here, we modified the pyrylium-scaffold to incorporate alkyne functionality to produce Raman reporters for the Raman silent region. We adapted our previously reported pyrylium dye synthesis procedure [17], which is based on reaction of $4 \mathrm{H}$-chalcogenopyranones with Grignard reagents to incorporate terminal alkynes (Scheme 1). In a similar manner, we adapted existing synthesis protocols of xanthone-based chromophores [18, 19] to incorporate alkyne- and cyano-functionality [20]. The newly established library of triple-bond containing pyrylium- and xanthylium dyes were subsequently evaluated for use as Raman reporters for the Raman silent region. Here, we report on the identification of several rationally designed Raman reporters that enable multiplexed SERS nanoparticle-based Raman imaging in the Raman silent region.

\section{Methods}

\section{Materials}

All chemicals were obtained from Sigma Aldrich

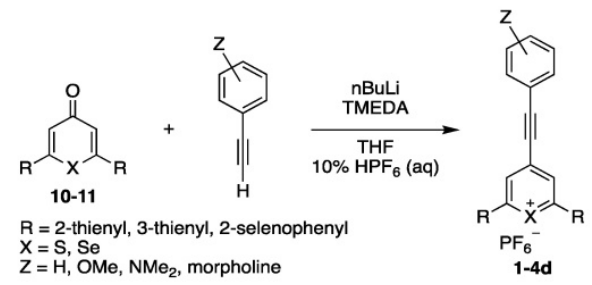

b

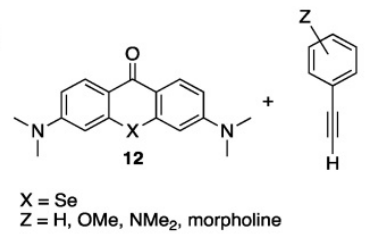

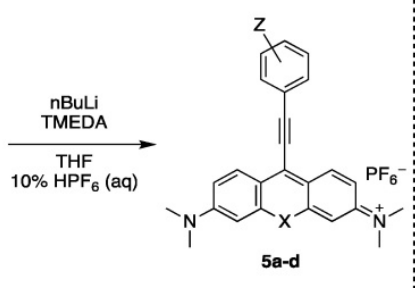

(St. Louis, MO) and were of the highest purity.

\section{General Synthesis of 4-ethynylphenyl substituted pyrylium or xanthylium dyes (1-5d)}

A flame-dried flask fit with a condenser under nitrogen was charged with a substituted phenylacetylene derivative $(0.830 \mathrm{mmol})$, tetramethylenediamine (TMEDA) (0.724 mmol), and anhydrous tetrahydrofuran (THF) $\left(3 \mathrm{~mL}\right.$ ) and cooled to $-78{ }^{\circ} \mathrm{C}$. To this solution, $n$-butyl lithium $(0.724 \mathrm{mmol})$ was added dropwise, and allowed to stir for $15 \mathrm{~min}$ at $-78^{\circ} \mathrm{C}$, and was subsequently warmed to ambient and stirred for $30 \mathrm{~min}$. This flask was subsequently cooled down to $-78^{\circ} \mathrm{C}$. In a separate flame-dried flask under nitrogen, the parent xanthone or pyranone $(0.361 \mathrm{mmol})$ was dissolved in THF $(3 \mathrm{~mL})$. The pyranone was transferred to the first flask via cannula. The resulting mixture was allowed to stir at $-78{ }^{\circ} \mathrm{C}$ for $15 \mathrm{~min}$, before being heated to $50{ }^{\circ} \mathrm{C}$ for $15 \mathrm{~min}$. This was subsequently cooled down to ambient temperature. The reaction mixture was then poured into $10 \%$ hexafluorophosphoric acid $(10 \mathrm{~mL})$. The mixture was filtered, the collected residue was dissolved in methylene chloride $\left(\mathrm{CH}_{2} \mathrm{Cl}_{2}\right)$ and dried with sodium sulfate. This was filtered, and the filtrate concentrated. The crude product was purified by a traditional recrystallization from boiling $\mathrm{CH}_{3} \mathrm{CN}$ and slowly cooled to yield the desired product.

\section{General Synthesis of Xanthylium-Cyano-Containing Dye (6-8)}

A flame-dried flask under nitrogen was charged with the parent xanthone $(0.168 \mathrm{mmol})$, and $\mathrm{CH}_{3} \mathrm{CN}$ $(8 \mathrm{~mL})$. To this solution, trifluoromethanesulfonic anhydride $(0.184 \mathrm{mmol})$ was added dropwise. After $30 \mathrm{~min}, \mathrm{KCN}(0.838 \mathrm{mmol})$ was added. After $3 \mathrm{~h}$, the reaction mixture was poured into a $10 \%$ solution of hexafluorophosphoric acid $(30 \mathrm{~mL})$. After stirring

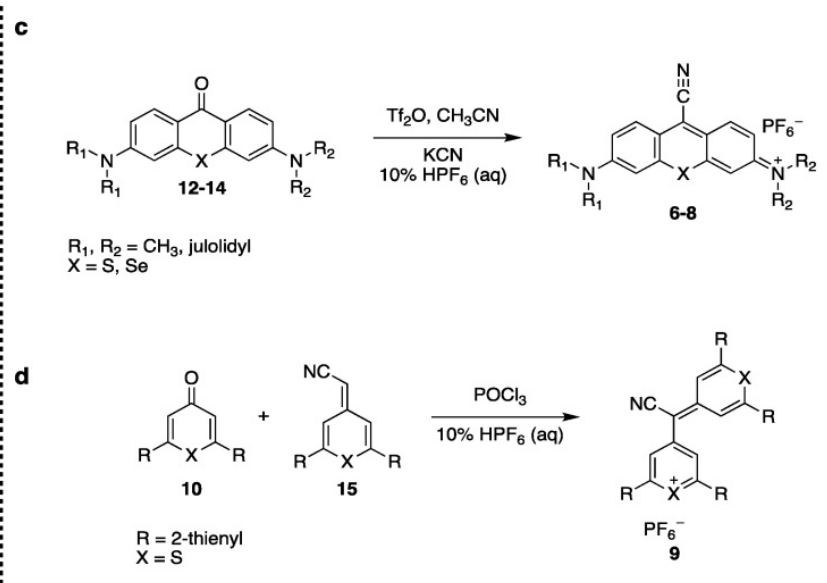


overnight, the mixture was filtered, and washed with water and ether $\left(\mathrm{Et}_{2} \mathrm{O}\right)$. The collected residue was dissolved in $\mathrm{CH}_{2} \mathrm{Cl}_{2}$ and dried with sodium sulfate. This was filtered, and the filtrate concentrated. The crude product was purified by a two-solvent recrystallization from $\mathrm{CH}_{2} \mathrm{Cl}_{2} / \mathrm{Et}_{2} \mathrm{O}$ to yield the desired chromophore.

\section{General Synthesis of Pyrylium-Cyano Dye (9)}

A flame-dried flask fit with a condenser under nitrogen was charged with the parent pyranone $(0.334$ $\mathrm{mmol})$ exocycliccyanomethylidene derivative (0.367 $\mathrm{mmol})$, and phosphorus oxychloride $(0.33 \mathrm{~mL})$. The reaction mixture was heated at reflux for $3 \mathrm{~h}$. The mixture was then cooled to ambient temperature and poured into a $10 \%$ hexafluorophosphoric acid solution $(20 \mathrm{~mL})$. The organic layer was extracted with methylene chloride (6 $15 \mathrm{~mL})$. The combined organic extracts were dried over sodium sulfate, filtered, and concentrated in vacuo. The residue was crystallized from acetonitrile $\left(\mathrm{CH}_{3} \mathrm{CN}\right)$. The solid was washed with hot portions of $\mathrm{CH}_{3} \mathrm{CN}$. The filtrate was concentrated, and the residual solid was purified by a traditional recrystallization from boiling $\mathrm{CH}_{3} \mathrm{CN}$ and slowly cooled to yield the desired chromophore.

\section{Raman spectroscopy}

All dyes were dissolved in dimethyl sulfoxide (DMSO) at a concentration of $30 \mathrm{mM}$ and added to 100 $\mathrm{L} /$ well $1.0 \mathrm{nM} 60-\mathrm{nm}$ gold nanoparticles in water to yield a final dye concentration of $300 \mathrm{M}$. After 1-min equilibration, surface-enhanced (resonance) Raman scattering spectra were acquired on a Horiba XplorRA+ Confocal Raman system using 638-nm and $785-\mathrm{nm}$ excitation lasers operating at $638 \mathrm{~nm}(30 \mathrm{~mW})$ and $785 \mathrm{~nm}(80 \mathrm{~mW})$ using a $1 \mathrm{~s}$ acquisition time and operating at $1 \%$ laser power.

\section{Results and Discussion}

\section{Raman reporter synthesis}

The triple bond containing pyrylium $(\mathbf{1 - 4}, \mathbf{9})$ or xanthylium dyes (5-8) were synthesized according to Scheme 1 (See Supplemental Information for detailed synthetic schemes). Ethynylphenyl-substituted pyrylium dyes (1-4d) were synthesized by terminally deprotonating the appropriate 4-ethynylphenyl derivative using n-butyl lithium (nBuLi) and subsequent addition to $4 \mathrm{H}$-chalcogenopyranone (Scheme 1a). The hexafluorophosphate salts were obtained by adding the associated alkynyl-substituted pyrylium dyes to $10 \%$ hexafluorophosphoric acid solution to yield chromophores 1-4d. As shown in Table 1, apart from the expected contribution of the ethynyl substitution to red-shifting the absorption maxima approximately $50 \mathrm{~nm}$ relative to analogous dyes [17], the absorption maxima were further red-shifted over a wide wavelength range by 1) substituting the chalcogen $(\mathrm{X})$ in the pyrylium core with a heavier chalcogen; 2 ) changing the substituents $(\mathrm{R})$ in the 2- and 6-position of the pyrylium core; and/or 3) by substituting the donor functionality (Z) on the phenyl-group [17]. The yields of the 4-ethynylphenyl-substituted pyrylium derivatives (1-4d) typically ranged from $15-33 \%$ (please see Supplemental Information).

Table 1. Structure and absorption maximum of 4-ethynylphenyl pyrylium derivatives.

\begin{tabular}{|c|c|c|c|c|c|c|}
\hline & Dye & Z & $x$ & $\mathrm{R}$ & $\lambda(\mathrm{nm})^{*}$ & $\log \varepsilon$ \\
\hline Z & 1 & $\mathrm{H}$ & $S$ & thiophen-3-yl & $411^{* * *}$ & 4.48 \\
\hline & 2 & $\mathrm{OMe}$ & $S$ & thiophen-2-yl & 525 & 4.81 \\
\hline & $3 a$ & morpholine & $S$ & thiophen-2-yl & $679 / 503$ & $4.70 / 4.40$ \\
\hline & $3 b$ & morpholine & $S$ & thiophen-3-yl & $592^{* *}$ & 4.54 \\
\hline & $3 c$ & morpholine & Se & thiophen-2-yl & $704 / 521$ & $4.73 / 4.45$ \\
\hline & $4 a$ & $\mathrm{NMe}_{2}$ & $S$ & thiophen-2-yl & $709 / 494$ & $4.86 / 4.38$ \\
\hline & $4 b$ & $\mathrm{NMe}_{2}$ & $S$ & thiophen-3-yl & 684 & 4.57 \\
\hline & $4 c$ & $\mathrm{NMe}_{2}$ & Se & thiophen-2-yl & $742 / 511$ & $4.76 / 4.30$ \\
\hline & $4 d$ & $\mathrm{NMe}_{2}$ & Se & selenophen-2-yl & $750 / 527$ & $4.87 / 4.42$ \\
\hline $\mathrm{F}_{6}^{-}$ & & & & & & \\
\hline
\end{tabular}

*Absorption spectra were measured of dye solution in dichloromethane unless otherwise noted. **acetonitrile

Xanthylium-based chromophores such as rhodamine 800 have been widely applied as Raman reporters [19, 21-24]. Inspired by previous work, we therefore synthesized a new class of cyano- and alkyne-containing structures, shown in Scheme 1b, c (See Supplemental Information for detailed synthetic schemes), generated from single xanthone-based precursors via nucleophilic attack at the 9-position of the xanthylium core. The incorporation of heavier chalcogen atoms induces sequential bathochromic shifts, favoring resonance enhancement at longer wavelengths. Additionally, constraining the nitrogen atom as seen in moving from the dimethyl amino xanthylium (dye 6) to the julolidine dyes (dyes 7,8 ) imparts a approximate $1020-\mathrm{nm}$ bathochromic shift [17]. This allows the absorption to be tuned towards the near-infrared region (Table 2 and 3). The yields for the 4-ethynylphenyl-substituted and cyanosubstituted xanthylium derivatives (6-8) ranged from $26-42 \%$ and $13-77 \%$, respectively (please see Supplemental Information).

Dye 9 was synthesized by emulating the work performed by VanAllan and coworkers [20], in which cyano-containing pyrylium dyes could be synthesized by allowing cyanoacetic acid to react with pyranones. The chalcogen tripod [25] was adapted in order to engender reporter molecules with higher affinity for typical SERS substrates such as gold or silver nanoparticles. 2,6-di(thiophen-2-yl)-4H-thiopyran-4one (10a) was treated with dimethyl sulfate, thus 
yielding 4-methoxy-2,6-di(thiophen-2-yl)thiopyrylium hexafluorophosphate (17) in 52\% yield [26]. A subsequent Knoevenagel condensation with cyanoacetic acid yielded 2-(2,6-di(thiophen-2-yl)-4Hthiopyran-4-ylidene)acetonitrile (15) in 67\% yield, containing both the desired chalcogen tripod and installed cyano group (See Scheme S1). As shown in Scheme 1d, the pyranone- and exocyclic cyanomethylidene precursors were subsequently condensed in phosphorus oxychloride, thusly forming dye 9 with an absorption maximum of 661 $\mathrm{nm}$.

Table 2. Structure and absorption maximum of alkynyl derivatives of selenoxanthylium dyes

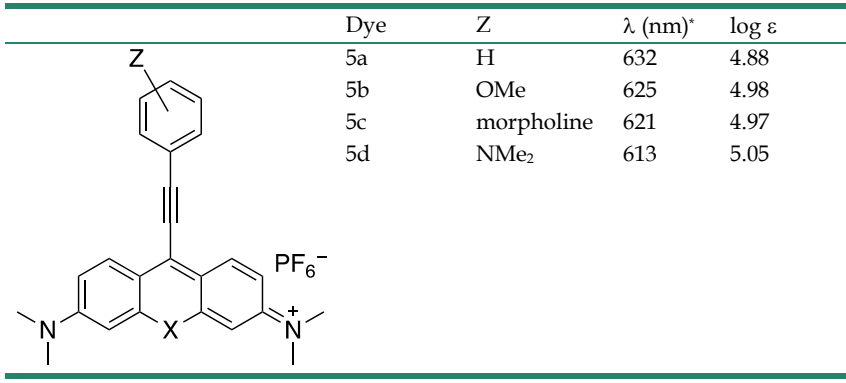

Table 3. Structure and absorption maximum of nitrile-derivatives of chalcogenoxanthylium dyes

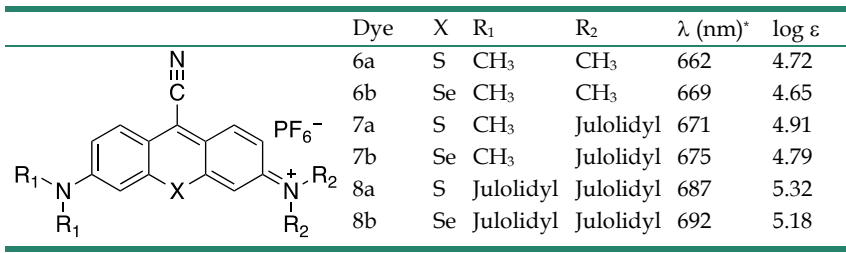

\section{Surface-enhanced Raman scattering measurements}

To assess and compare the performance of the newly synthesized dyes, the dyes were grouped into four groups: the ethynyl-substituted chalcogenopyrylium dyes (1-4); ethynyl-substituted- (5a-d); and nitrile-substituted xanthylium dyes (6-8), and the cyanopyrylium dye (9). The dyes were dissolved in dimethylsulfoxide (DMSO) to yield a concentration of $10 \mathrm{mM}$ and added to a $100-\mu \mathrm{l}$ dispersion of $60-\mathrm{nm}$ gold nanoparticles $(1.0 \mathrm{nM})$ in water to yield a final dye concentration of $100 \mu \mathrm{M}$. We selected $60-\mathrm{nm}$ spherical gold nanoparticles, because those are the most widely-applied size and shape in biomedical applications [6-8, 27-32], and were previously shown to generate the strongest SERS enhancements [33]. The SERS spectra were measured in the range of $300-3000 \mathrm{~cm}^{-1}$ on a Horiba XploRA+ confocal Raman microscope equipped with 532-nm, 638-nm, and $785-\mathrm{nm}$ excitation lasers. However, here we will only present the SERS spectra after excitation with 638- and $785-\mathrm{nm}$ lasers, because it was found that 532-nm excitation did not produce any appreciable SERS (even at maximum power of $80 \mathrm{~mW}$ ). Based on theoretical considerations [34], a contribution to the SERS enhancement from the $60-\mathrm{nm}$ gold nanoparticle core was expected, since 532-nm is close to the local surface plasmon resonance of $548 \mathrm{~nm}$ of the unmodified 60-nm gold nanoparticles.

As shown in Fig. 1, 638-and 785-nm excitation of ethynyl-substituted pyrylium dyes 1a-4 led to Raman bands $\left(\sim 2100-2200 \mathrm{~cm}^{-1}\right)$ in the Raman silent region $\left(1750-2750 \mathrm{~cm}^{-1}\right)$. The Raman shifts of the alkyne match to those previously reported for internal alkynes [16]. The dimethylamino-derivatives (4a and 4d) in particular produced Raman bands with high intensities ( 3000 cps), relative to the other dyes ( $<1000$ cps). Substitution of the chalcogen atom in the pyrylium ring from sulfur to selenium shifted the Raman band associated with the ring-breathing mode of the aromatic pyrylium ring from $1550-1560 \mathrm{~cm}^{-1}$ to $1570-1590 \mathrm{~cm}^{-1}$ (depending on the excitation laser wavelength), and also shifted the Raman band associated with the ethynyl stretching mode from $2120-2140 \mathrm{~cm}^{-1}$ for the thiopyrylium dyes to 2140 $2170 \mathrm{~cm}^{-1}$ for the selenopyrylium dyes [16]. Furthermore, Yamakoshi et al. reported that substituents at the 4-position on the phenyl ring enhanced intensities by extending the $\pi$-orbitals in the direction of the alkyne stretching [16]. We also found that changing the functionality at the 4-phenyl markedly altered the Raman shift and intensities of the alkyne stretching modes of the chalcogenopyrylium dyes for methoxyor dimethylamino-substituents relative to a morpholino-substituted phenyl ring or non-substituted phenyl ring (vide supra). We further explored this effect of the substituents on the Raman shift of the ethynyl stretching mode in the ethynyl-substituted xanthylium dyes (5a-d) that contained different substituents on the phenyl ring adjacent to the ethynyl substituent. We found that the functional groups affected the Raman shift of the ethynyl stretching mode and depending on the substituent induced redor blue shifts of $10 \mathrm{~cm}^{-1}$ relative to the non-substituted phenyl ring (Fig. 2). The substituents in the 4-position on the phenyl ring induced a shift in the following order for the alkyne stretching mode from $\mathrm{NMe}_{2}<$ morpholine $<\mathrm{OMe}<\mathrm{H}$, ranging from 2150 up to 2200 $\mathrm{cm}^{-1}$, respectively. However, we did not observe the same effect on intensity of the alkyne stretching mode of the substituted xanthylium dyes, as observed for the chalcogenopyrylium dyes. 

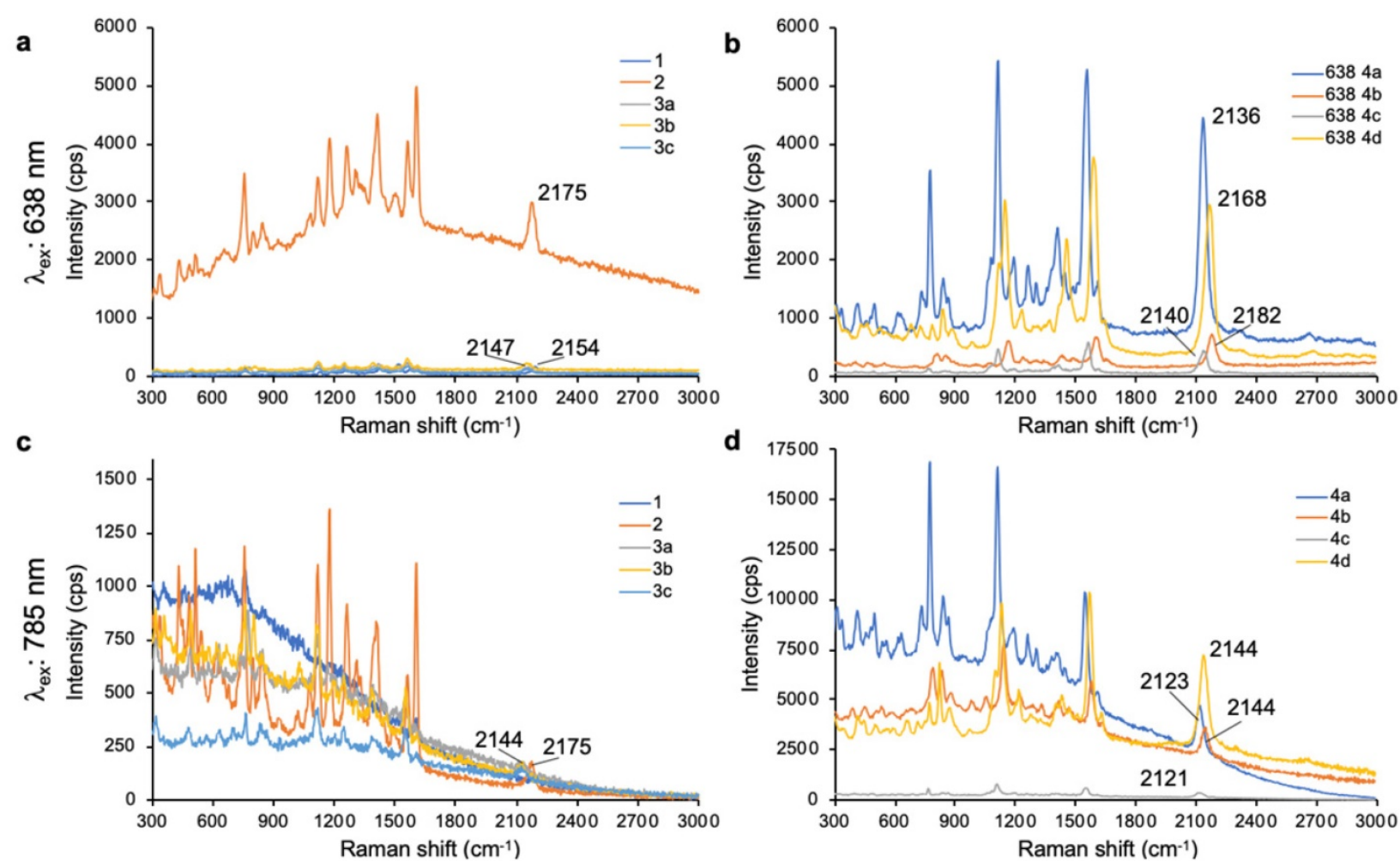

Figure 1. Surface-enhanced Raman scattering spectra of 4-ethynylphenyl substituted pyrylium dyes (1-4d). (a) SERS spectra of pyrylium dyes 1-3c after 638-nm excitation. (b) SERS spectra of pyrylium dyes 4a-d after 638-nm excitation. (c) SERS spectra of pyrylium dyes 1-3c after 785-nm excitation. d) SERS spectra of pyrylium dyes 4a-d after 785-nm excitation.

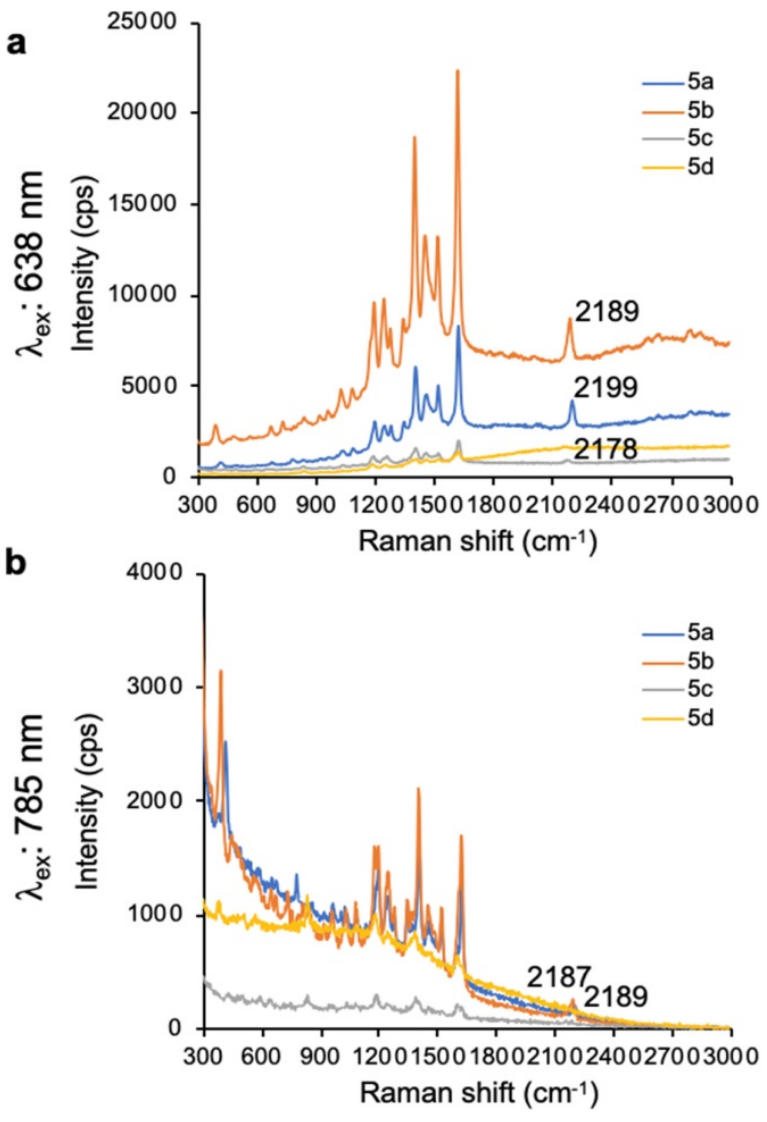

Figure 2. SERS spectra of dyes 5 a-d upon 638-nm (a) and 785-nm (b) laser excitation.
In addition to ethynyl substituted xanthylium dyes, we explored cyano-substituted xanthylium and mono- and bisjulolidyl extended xanthylium dyes as Raman reporters for the Raman silent region (Table 3 ). The two julolidyl moieties impart added rigidity and bathochromic shifts in the wavelength of maximum absorption of the resulting dyes of $\sim 20$ nm.[35] As shown in Figure 3, the cyano-substituted selenoxanthylium dyes produced a Raman band in the Raman-silent region around $2230 \mathrm{~cm}^{-1}$, while the cyano-substituted thioxanthylium and mono-julolidyl extended thioxanthylium dyes produced strong fluorescence upon 638-nm excitation. Upon 785-nm excitation, which is $\sim 100 \mathrm{~nm}$ removed from the excitation maxima of the dyes (Table 3; Fig. 3), the fluorescence background of all cyano-substituted xanthylium dyes is markedly reduced and all dyes produce a Raman-band in the Raman-silent region albeit with a relatively weak intensity (i.e. $\sim 3$-fold reduction) relative to the intensity produced by dye $\mathbf{6 b}$ and $\mathbf{7 b}$ after $638-\mathrm{nm}$ excitation.

Lastly, we evaluated dye 9 where the cyano-functionality was introduced within the conjugated methine system. As shown in Fig. 4, dye 9 produced a weak Raman band at $2383 \mathrm{~cm}^{-1}$ after 638-nm laser excitation; a red-shift of an additional $150 \mathrm{~cm}^{-1}$ relative to the Raman band produced by cyano-substituted dyes 6-8. The intensity of the 
Raman band of $-\mathrm{C} \equiv \mathrm{N}$ was too weak to be observed upon 785-nm laser excitation. Possibly, dye 9 is unstable in an aqueous environment and decomposed. We therefore abandoned dye 9.

Pyrylium and xanthylium-based dyes represent some of the most sensitive Raman reporters synthesized to date $[9,11,29]$. One aim of this work was to generate novel reporters combining the properties that enable high sensitivity, along with functional groups exhibiting vibrational fingerprints in the Raman silent region. As shown in Fig. 5, we selected dye $\mathbf{4 a}, \mathbf{4 b}, \mathbf{4 d}, \mathbf{5 a}, \mathbf{5 b}$, and $\mathbf{6 b}$ as promising Raman reporters for SERS applications using 638-nm laser excitation (Fig. $\mathbf{5 b - c}$ ) and dyes $\mathbf{4 a}, \mathbf{4 b}, \mathbf{4 d}$ and $\mathbf{6 b}$ for applications using 785-nm excitation (Fig. 5d-e). We excluded dyes $\mathbf{5 a}$ and $\mathbf{5 b}$ as candidates for $785-\mathrm{nm}$ because the intensities of the Raman bands in the Raman-silent region were too low.

The Raman reporters described here should be readily distinguishable from previously synthesized reporters that demonstrated intense pyryliumbreathing modes at or around $1600 \mathrm{~cm}^{-1}$. This was accomplished by incorporating both -alkyne and cyano functional groups into chromophore structures. In the current study, we have demonstrated the synthesis of novel Raman reporter molecules that are capable of displaying resonance enhancement with a 638-nm and 785-nm excitation source and displaying Raman fingerprints in the Raman silent region. It was demonstrated that the di-selenophen-2-yl selenopyrylium dye $\mathbf{4 d}$ exhibited remarkable sensitivity using a $785-\mathrm{nm}$ excitation source. This is likely due to the absorbance of the dye closely overlapping with the excitation source that also has a large extinction coefficient, permitting efficient resonance enhancement to occur. Our work corroborated the findings by Yamakoshi et al.[16] in which the Raman shift displayed by reporter molecules was very sensitive to substituents on the para-substituted aromatic rings on the chromophore. Since the stretching frequency is proportional to the square root of the bond strength divided by the reduced mass [19], stronger electron donating substituents may lead to more cumulene-like behavior [36], weakening bond strength and therefore lowering the stretching frequency. This is evident when one compares the phenylacetylene-substituted xanthylium dye 5a to the analogous anisole derivative dye $5 \mathbf{b}$. Using the 638-nm excitation source, six Raman bands can be individually identified. This decreased to four when the 785-nm excitation source is used, likely due to minimal resonant enhancement of dyes $\mathbf{5 a}$ and $\mathbf{5 b}$ at this wavelength.
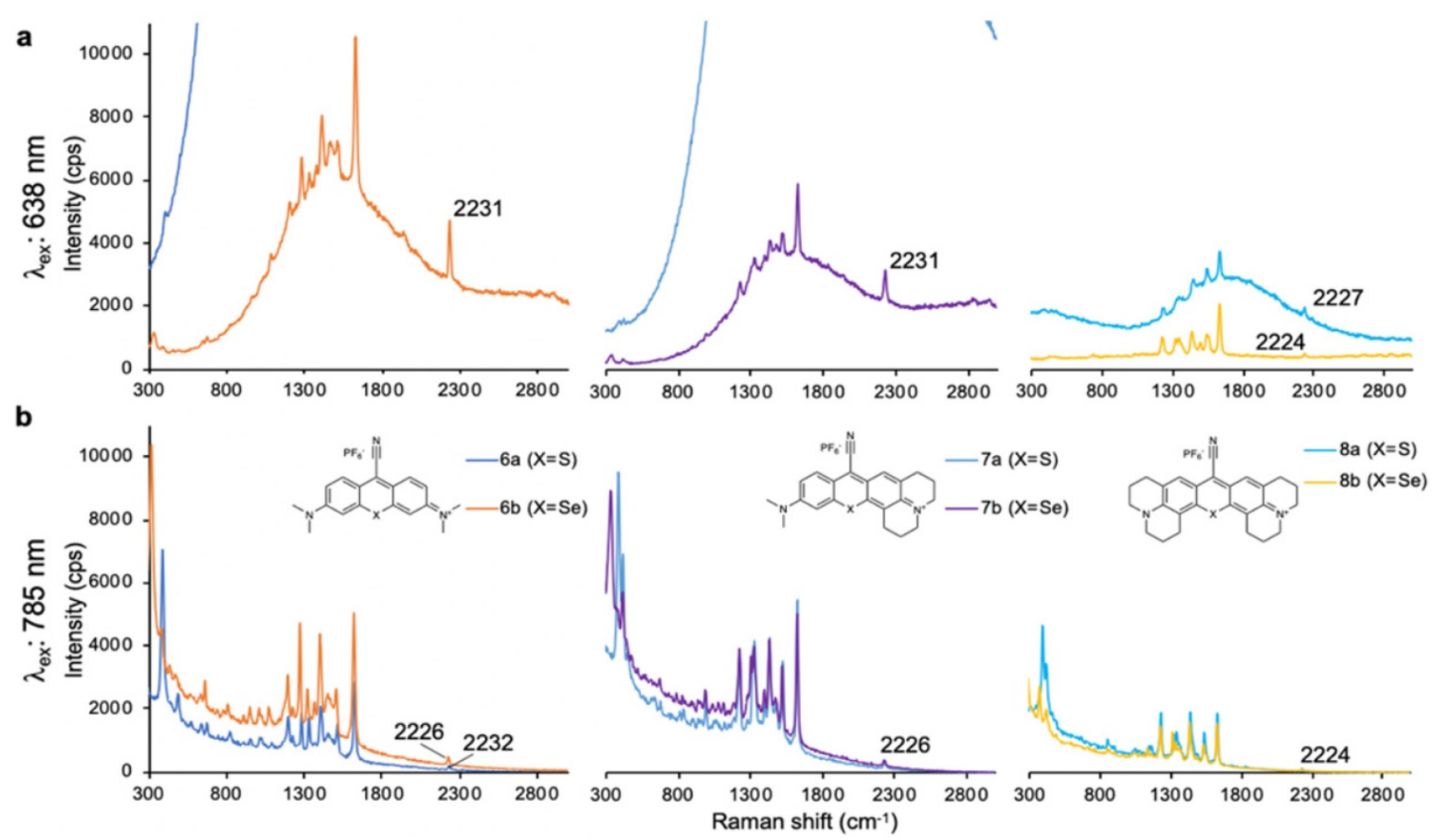

Figure 3. SERS spectra of cyano-substituted thio- (6a-8a) and selenoxanthylium (6b-8b) dyes upon 638-nm (a) and 785-nm (b) laser excitation 

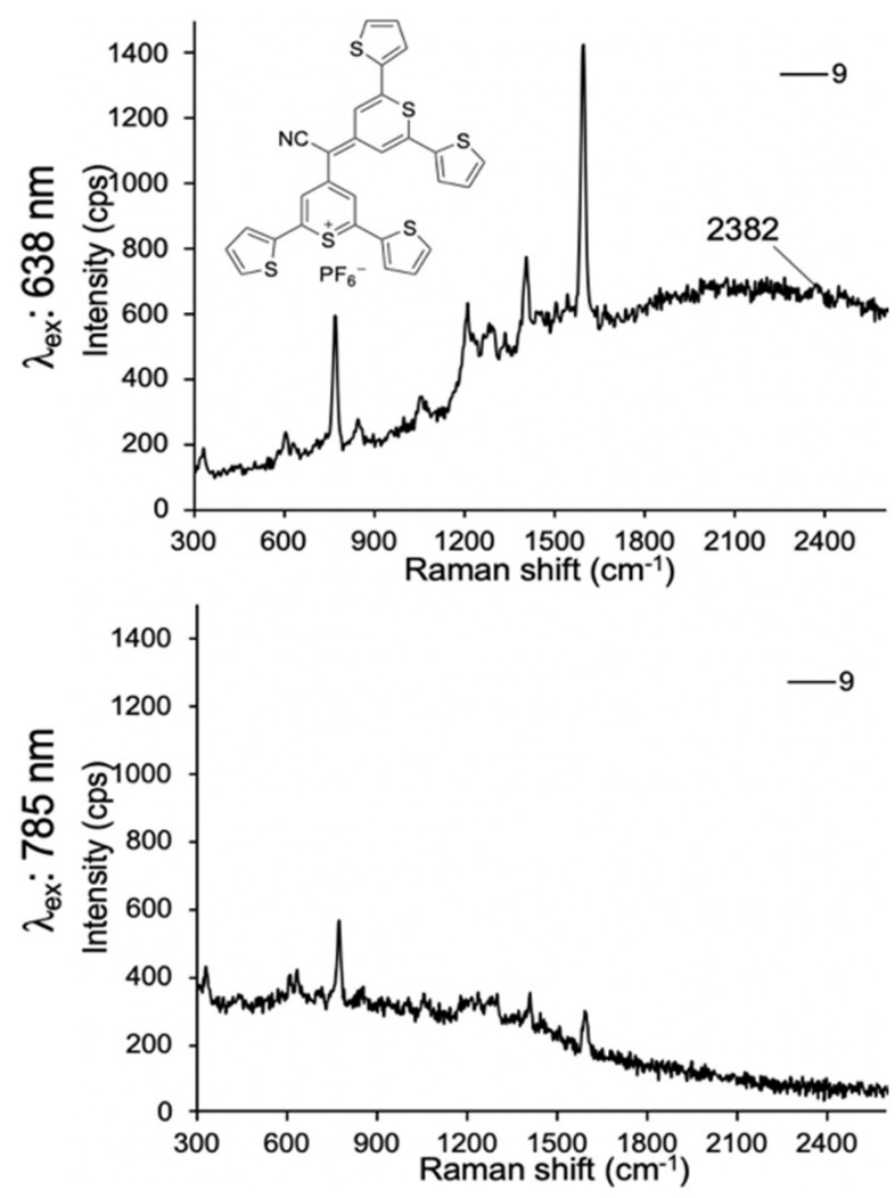

Figure 4. SERS spectra of dye 9 upon 638-nm (a) and 785-nm (b) laser excitation.

a

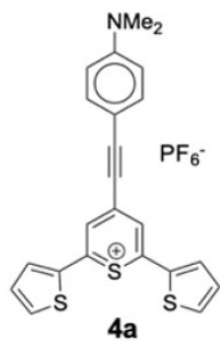

4a<smiles>CN(C)c1ccc2c(C=Cc3ccccc3)c(C(F)(F)F)ccc2c1</smiles>

$5 a$

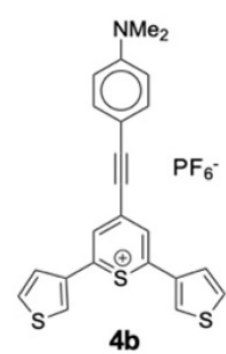

$4 \mathrm{~b}$<smiles></smiles>

$5 b$

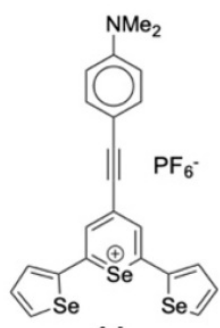

4d

$6 b$
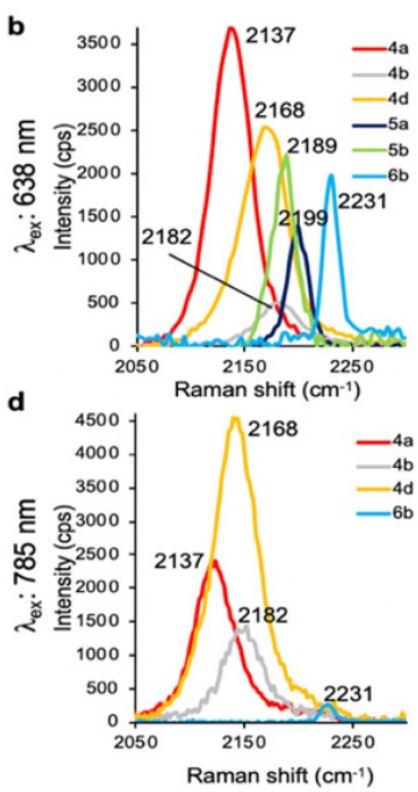
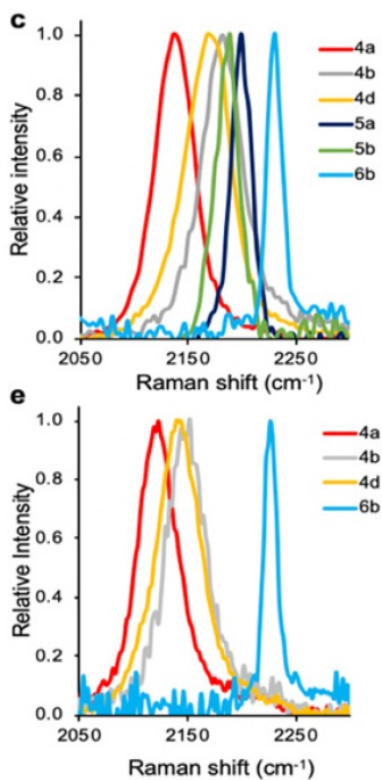

Figure 5. Raman bands in the Raman-silent region. a, Structures of the selected resonant Raman reporters with the most intense Raman bands in the silent region. b, Composite of measured Raman intensity, and c, relative Raman intensity upon 638-nm laser excitation. d, Composite of measured Raman intensity, and e, Relative Raman intensity upon 785-nm laser excitation.

\section{Conclusions}

We have designed, synthesized, and evaluated alkyne- and cyano-substituted chalcogenopyrylium and xanthylium dyes as Raman reporters for the Raman-silent region. We identified 6 candidates that can be used as Raman reporters for SERS applications using 638-nm excitation and 4 candidates for use with 
a 785-nm excitation source. Moreover, we identified dye $4 \mathrm{~d}$ that upon 785-nm laser excitation produced a high-intensity Raman band in the Raman-silent region where Raman background of tissues is minimized. As such, dye $\mathbf{4 d}$ holds great promise as a Raman reporter for a stand-alone SERS-based Raman imaging probe for detection of biomarkers in vivo as well.

\section{Abbreviations}

$\mathrm{CH}_{2} \mathrm{Cl}_{2}$ : methylene chloride; $\mathrm{CH}_{3} \mathrm{CN}$ : acetonitrile; DMSO: dimethylsulfoxide; $\mathrm{Et}_{2} \mathrm{O}$ : diethylether; $\mathrm{KCN}$ : potassium cyanide; SERS: surface-enhanced Raman scattering; THF: tetrahydrofuran; TMEDA: tetramethylenediamine.

\section{Supplementary Material}

Supplementary synthesis procedures.

http://www.ntno.org/v06p0001s1.pdf

\section{Acknowledgements}

This work was supported in part by the following grants: NCI 1R01 CA182043-01A1 (S.S.G.), NCI CCNE U54 (S.S.G.). Part of this work was performed at the Stanford Nano Shared Facilities (SNSF), supported by the National Science Foundation under award ECCS-1542152.

\section{Competing Interests}

Stefan Harmsen is listed as inventor on several patents related to the SERS technology used in this work. Sanjiv Sam Gambhir holds the original patent on the use of SERS in living subjects.

\section{References}

1. McFarland AD, Young MA, Dieringer JA, Van Duyne RP. Wavelength-Scanned Surface-Enhanced Raman Excitation Spectroscopy. J Phys Chem B. 2005; 109: 11279-85.

2. Zavaleta CL, Smith BR, Walton I, Doering W, Davis G, Shojael B, et al. Multiplexed imaging of surface enhanced Raman scattering nanotags in living mice using noninvasive Raman spectroscopy. Proc Natl Acad Sci US A. 2009; 106: 13511-6, S/1-S/4.

3. Dinish US, Yaw FC, Agarwal A, Olivo M. Development of highly reproducible nanogap SERS substrates: Comparative performance analysis and its application for glucose sensing. Biosens Bioelectron. 2011; 26: 1987-92.

4. Laing S, Gracie K, Faulds K. Multiplex in vitro detection using SERS. Chem Soc Rev. 2016; 45: 1901-18.

5. Wachsmann-Hogiu S, Weeks T, Huser T. Chemical analysis in vivo and in vitro by Raman spectroscopy-from single cells to humans. Curr Opin Biotechnol. 2009; 20: 63-73.

6. Harmsen S, Huang R, Wall MA, Karabeber H, Samii JM, Spaliviero M, et al. Surface-enhanced resonance Raman scattering nanostars for high-precision cancer imaging. Sci Transl Med. 2015; 7: 271ra7.

7. Harmsen S, Rogalla S, Huang R, Spaliviero M, Neuschmelting V, Hayakawa Y, et al. Detection of Pre-Malignant Gastrointestinal Lesions Using Surface-Enhanced Resonance Raman Scattering-Nanoparticle Endoscopy. ACS Nano. 2019

8. Huang R, Harmsen S, Samii JM, Karabeber H, Pitter KL, Holland EC, et al. High Precision Imaging of Microscopic Spread of Glioblastoma with a Targeted Ultrasensitive SERRS Molecular Imaging Probe. Theranostics. 2016; 6: 1075-84.

9. Bedics MA, Kearns H, Cox JM, Mabbott S, Ali F, Shand NC, et al. Extreme red shifted SERS nanotags. Chem Sci. 2015; 6: 2302-6.
10. Kearns H, Ali F, Bedics MA, Shand NC, Faulds K, Detty MR, et al. Sensitive SERS nanotags for use with a hand-held $1064 \mathrm{~nm}$ Raman spectrometer. R Soc Open Sci. 2017; 4: 170422/1-/9.

11. Kearns H, Bedics MA, Shand NC, Faulds K, Detty MR, Graham D. Sensitive SERS nanotags for use with $1550 \mathrm{~nm}$ (retina-safe) laser excitation. Analyst (Cambridge, U K). 2016; 141: 5062-5.

12. Moreton S, Faulds K, Shand NC, Bedics MA, Detty MR, Graham D. Functionalisation of hollow gold nanospheres for use as stable, red-shifted SERS nanotags. Nanoscale. 2015; 7: 6075-82.

13. Huang NY, Short M, Zhao JH, Wang HQ, Lui H, Korbelik M, et al. Full range characterization of the Raman spectra of organs in a murine model. Opt Express. 2011; 19: 22892-909.

14. Surmacki J, Musial J, Kordek R, Abramczyk H. Raman imaging at biological interfaces: applications in breast cancer diagnosis. Mol Cancer. 2013; 12.

15. Yamakoshi H, Dodo K, Okada M, Ando J, Palonpon A, Fujita K, et al. Imaging of EdU, an Alkyne-Tagged Cell Proliferation Probe, by Raman Microscopy. J Am Chem Soc. 2011; 133: 6102-5.

16. Yamakoshi H, Dodo K, Palonpon A, Ando J, Fujita K, Kawata S, et al. Alkyne-tag Raman imaging for visualization of mobile small molecules in live cells. J Am Chem Soc. 2012; 134: 20681-9.

17. Detty MR, McKelvey JM, Luss HR. Tellurapyrylium dyes. 2. The electron-donating properties of the chalcogen atoms to the chalcogenapyrylium nuclei and their radical dications, neutral radicals, and anions. Organometallics. 1988; 7: 1131-47.

18. Kryman MW, Schamerhorn GA, Hill JE, Calitree BD, Davies KS, Linder MK, et al. Synthesis and Properties of Heavy Chalcogen Analogues of the Texas Reds and Related Rhodamines. Organometallics. 2014; 33: 2628-40.

19. Wei L, Chen Z, Shi L, Long R, Anzalone AV, Zhang L, et al. Super-multiplex vibrational imaging. Nature (London, U K). 2017; 544: 465-70.

20. VanAllan JA, Reynolds GA, Maier DP. Reactions of cyanoacetic acid with 2,6-diphenyl-4-pyrone. J Org Chem. 1968; 33: 4418-21.

21. Etchegoin PG, Le Ru EC, Meyer M. Evidence of natural isotopic distribution from single-molecule SERS. J Am Chem Soc. 2009; 131: 2713-6.

22. Le Ru EC, Blackie E, Meyer M, Etchegoin PG. Surface Enhanced Raman Scattering Enhancement Factors: A Comprehensive Study. J Phys Chem C. 2007; 111: 13794-803.

23. Michaels AM, Nirmal M, Brus LE. Surface Enhanced Raman Spectroscopy of Individual Rhodamine 6G Molecules on Large Ag Nanocrystals. J Am Chem Soc. 1999; 121: 9932-9.

24. Nie S, Emory SR. Probing single molecules and single nanoparticles by surface-enhanced Raman scattering. Science (Washington, D C). 1997; 275: 1102-6.

25. Kearns H, Sengupta S, Sasselli IR, Bromley Iii L, Faulds K, Tuttle T, et al. Elucidation of the bonding of a near infrared dye to hollow gold nanospheres - a chalcogen tripod. Chem Sci. 2016; 7: 5160-70.

26. Chen $\mathrm{CH}$, Reynolds GA, Van Allan JA. Synthesis of 4H-thiopyran-4-ones. J Org Chem. 1977; 42: 2777-8.

27. Neuschmelting V, Harmsen S, Beziere N, Lockau H, Hsu HT, Huang R, et al. Dual-Modality Surface-Enhanced Resonance Raman Scattering and Multispectral Optoacoustic Tomography Nanoparticle Approach for Brain Tumor Delineation. Small. 2018; 14: e1800740.

28. Pal S, Harmsen S, Oseledchyk A, Hsu HT, Kircher MF. MUC1 Aptamer Targeted SERS Nanoprobes. Adv Funct Mater. 2017; 27.

29. Harmsen S, Bedics MA, Wall MA, Huang R, Detty MR, Kircher MF. Rational design of a chalcogenopyrylium-based surface-enhanced resonance Raman scattering nanoprobe with attomolar sensitivity. Nat Commun. 2015; 6: 6570.

30. Harmsen S, Wall MA, Huang R, Kircher MF. Cancer imaging using surface-enhanced resonance Raman scattering nanoparticles. Nat Protoc. 2017; 12 : 1400-14.

31. Kircher MF, de la Zerda A, Jokerst JV, Zavaleta CL, Kempen PJ, Mittra E, et al. A brain tumor molecular imaging strategy using a new triple-modality MRI-photoacoustic-Raman nanoparticle. Nat Med. 2012; 18: 829-34.

32. Spaliviero M, Harmsen S, Huang R, Wall MA, Andreou C, Eastham JA, et al. Detection of Lymph Node Metastases with SERRS Nanoparticles. Mol Imaging Biol. 2016; 18: 677-85.

33. Hong SM, Li X. Optimal Size of Gold Nanoparticles for Surface-Enhanced Raman Spectroscopy under Different Conditions. J Nanomater. 2013.

34. Stiles PL, Dieringer JA, Shah NC, Van Duyne RP. Surface-enhanced Raman spectroscopy. Annu Rev Anal Chem. 2008; 1: 601-26.

35. Shi I, Zhang X-P, Neckers DC. Xantheses: Fluorone Derivatives II. Tetrahedron Lett. 1993; 34: 6013-6. 
36. Akiyama S, Nakatsuji S, Nakashima K, Watanabe M, Nakazumi H. Triphenylmethane dye ethynologs with absorption bands in the near infrared. J Chem Soc, Perkin Trans 1. 1988: 3155-61. 